Journal of Computer Science 7 (11): 1619-1625, 2011

ISSN 1549-3636

(C) 2011 Science Publications

\title{
Identification of Electrooculography Signals Frequency Energy Distribution Using Wavelet Algorithm
}

\author{
${ }^{1}$ W.M. Bukhari, W. Daud, ${ }^{2}$ Rubita Sudirman and ${ }^{3}$ Camallil Omar \\ ${ }^{1}$ Faculty of Engineering and Technology, University of Multimedia, Malacca \\ ${ }^{2-3}$ Faculty of Electrical Engineering, Universiti Teknologi Malaysia \\ 81310 UTM Johor Bahru, Johor, Malaysia
}

\begin{abstract}
Problem statement: The time frequency analysis of non-stationary signals has been the considerable research effort in recent years. Wavelet transform is one of the favored tool for the analyzing the biomedical signals. Approach: We describe the identification of ElectroOculograph (EOG) signals of eye movement potentials by using wavelet algorithm which gives a lot of information than FFT. The capability of wavelet transform was to distribute the signal energy with the change of time in different frequency bands. This will showed the characteristic of the signals since energy was an important physical variable in signal analysis. The EOG signals were captured using electrodes placed on the forehead around the eyes to record the eye movements. The wavelet features used to determine the characteristic of eye movement waveform. This technique adopted because it was a non-invasive, inexpensive and accurate. The new technology enhancement has allowed the EOG signals captured using the Neuronal EEG-9200. The recorded data was composed of an eye movement toward four directions, i.e., downward, upward, leftward and rightward. The proposed analysis for each eyes signal is analyzed by using Wavelet Transform (WT) with energy algorithm and by comparing the energy distribution with the change of time and frequency of each signal. Results: A wavelet Scalogram was plotted to display the different percentages of energy for each wavelet coefficient towards different movement. Conclusion: From the result, it is proved that the different EOG signals exhibit differences in signals energy with their corresponding scale such as leftward with scale 6 (8$16 \mathrm{~Hz})$, rightward with scale $8(2-4 \mathrm{~Hz})$, downward with scale $9(1-2 \mathrm{~Hz})$ and upward with level 7 $(4-8 \mathrm{~Hz})$. Statistically, the results in this study indicate that there are $93 \%$ (averages) significance differences in the extracted features of wavelet Scalogram analysis.
\end{abstract}

Key words: Eye movement, signal potentials, wavelet transform, energy distribution, Artificial Neural Network (ANN), Data Acquisition System (DAS), daubechies order, biomedical instrumentation, frequency analysis

\section{INTRODUCTION}

The human eye is a spherical structure with a radius of $12 \mathrm{~mm}$. The signal potentials by Rushaidin et al. (2009), is a signals that can be sense from the movement of the human eyes can be known as Electrooculograph (EOG). EOG is the electrical recording corresponding to the eye movement. The eye has a resting electrical potential, with the front of the globe positive and back with globe negative. This phenomenon was first observed by Emil du BoisReymond in 1848 and has been the foundation in electrooculography as discussed by Malmivuo and Plonsey (1995).

EOG are taken using bipolar electrodes on the outside of eye. Exact electrode placements vary, but the electrode generally placed on the temples or on the distal ends of the forehead. When the eyes move, a differential potential result will occur. The magnitudes of the right and left eye movements as stated by Choo and Somnuk (2009), it can be seen between -75-150uV respectively. Malmivuo and Plonsey (1995) also stated that the polarity of movement potentials is dependent on electrode setup since the signal is positive when the eyes are moving toward positive electrode.

EOG is a technique for measuring the resting potential of the retina. The resulting signal is called the electro-oculogram. The main applications are in ophthalmological diagnosis and in recording eye movements stated by Jagla et al. (2007) writes that EOG is a potential produced by movement of the eye or eye lid. Nowadays, the recording of the EOG is a routinely applied diagnostic method for investigating the human oculomotor system, such as in sleep studies as proved by Lusted et al., (1993). This EOG signal is picked up by a bi-channel signal acquisition system consisting of the Horizontal $(\mathrm{H})$ and Vertical (V) channels.

Corresponding Author: W.M. Bukhari, Faculty of Engineering and Technology, University of Multimedia, Malacca Malaysia, Tel: +606 2523007 
At present, EOG is used for the evaluation of occulomotor abnormalities such as nystagmus, strabismus and supranuclear occulomotor dysfunction is briefly explained by Shinomiya et al. (2008). Besides, Kherlopian et al. (2006) stated that EOG signals can be used in various aspect of medical and biomedical application beyond diagnostics, for instance, in assisting the disabled. Numerous other techniques from theory of biomedical signal processing have been used to obtain representations and extract the features of interest for classification purposes. Kumar and Poole (2002) used the EOG signals for determining the angle of eye gaze for controlling a computer while Guven and Kara (2006) used the EOG signals for the classification with Artificial Neural Network (ANN) and Sudirman and Bukhari (2009) used the eye movement for the classification by using time frequency analysis. There has been a number of attempts have been made by the engineers and researchers to process non-stationary signals such as EOG in an appropriate way in order to circumvent the disadvantages of the Fourier Transform. Elhelw et al. (2008) used discrete time Markov chains to investigate the temporal fixation sequences. However, the study involved the Wavelet Transform of EOG signals from the previous research only emphasized on the noise elimination or the signal compression.

This study is presented as follows. Section 1 briefly gives an introduction to Wavelet Transform (WT) as discussed by Rizon (2010) along with the wavelet scalogram. Section 2 focused on the methodology and approaches related to the study. In section 3, the study briefly discuss about the result from the study. In section 4, conclusion is drawn to summarize the whole study and concludes the result of the identification.

\section{Wavelet transforms:}

Background: Wavelet transform is a powerful tool in the analysis of signals because of its ability to extract time and requency information. Hazarika et al. (1997) in his work informed that the wavelet transform could be defined as an extension of the classic Fourier transform, except that, instead of working on a single scale (time frequency)it works on a single scale basis.

Wavelet functions overcome the limitations of Fourier methods by employing analyzing function that are local in time and frequency. It is a finite energy function and can be represented on a transient signals. In signal processing, wavelet analysis is importantly used in processing the non-stationary signals. The wavelet transform can be interpreted as a decomposition of the original signal into set of independent frequency channel. It is essentially the method of processing data from a continuous signal into series of signal decomposition represented at different frequency ranges. This is the used of mother wavelet. The window function of wavelet transform can be defined as, $\psi_{\mathrm{ab}}(\mathrm{t})$ : (Eq. 1)

$$
\psi_{\mathrm{ab}}(\mathrm{t}) \stackrel{\Delta}{=} \frac{1}{\sqrt{\mathrm{a}}} \Psi\left(\frac{\mathrm{t}-\mathrm{b}}{\mathrm{a}}\right)
$$

Where:

$\mathrm{a}=$ Scale

$\mathrm{b}=$ Translation

If $\mathrm{a}$ and $\mathrm{b}$ is 1 , the equation is known as the basic or mother wavelet. It can be used to generate other wavelet at different scales and translation. The larger a will result on wider window in time domain and smaller width in frequency domain or vice versa. This is because of the dependences of time and frequency resolution on scale, a. The scale parameter $a$ is defined as $\mid 1 /$ frequency| and corresponds to frequency information. Scaling either dilates (expands) or compresses a signal.

Wavelet also can be found in biomedical signal processing. In this research study, wavelet transform is used in order to represent the captured signals of EOG for their significant features. Meanwhile, yourself 2006 proposed in his effort on wavelet transform that this method enables discrimination between no stationary signals exhibiting different frequency. From the features observed in the wavelet transform implementation, it is hope that the identification of the different types of EOG signals could be derived specifically. It might be represented either in frequency or time domain of energy distribution of the signals.

Wavelet scalogram: Wavelet Scalogram communicates the time frequency localization property of the wavelet transform. In this plot each detail coefficient is plotted as a filled rectangle whose color corresponds to the magnitude of the coefficient. The location and size of the rectangle are related to the time interval and the frequency range for this coefficient. Coefficients at low levels are plotted as wide and short rectangles to indicate that they localize a wide time interval but a narrow range of frequencies in the data. In contrast, rectangles for coefficients at high levels are plotted thin and tall to indicate that they localize small time ranges but large frequency ranges in the data. The heights of the rectangles grow as a power of 2 as the level increases.

\section{MATERIALS AND METHODS}

Experimental setup: This system setting includes the EEG Data Acquisition System (DAS) Rahmat et al. (2010); Neuronal 9200 software (Fig. 1-2) with EOG electrodes set (Fig. 1-4) and the sampling interval is $1 \mathrm{~ms}$. The EEG data acquisition system is used to record EOG signals from the subjects. Independent measurements can be obtained from both 
eyes, but as both eyes move in the vertical direction, it is sufficient to measure the vertical motion of only one eye together with the horizontal motion of both eyes. $\mathrm{Ag} / \mathrm{AgCl}$ electrodes (Fig. 1-3) are chosen as their half cell potential is closer to zero compared to other types such as silicon rubber electrodes.

This process was done in a quiet room to minimize the noise and hence get better recorded signals as shown in (Fig. 2). Subjects were seated on a chair and supervised by an instructor who gave instructions on how to move their eyes as shown on Fig. 1-4. The instruction composed of four movements that are downward, upward, left and right. The recording was done in four successive eye movements for 15 subjects and each subject repeated for three times. Initially, EOG was recorded for 20 to 30 seconds for each eyes movement. Unfortunately, since the subjects were showing signs of tiredness, the recording duration has been reduced to 10 seconds which was free from artefacts observed in longer traces by visual inspection.

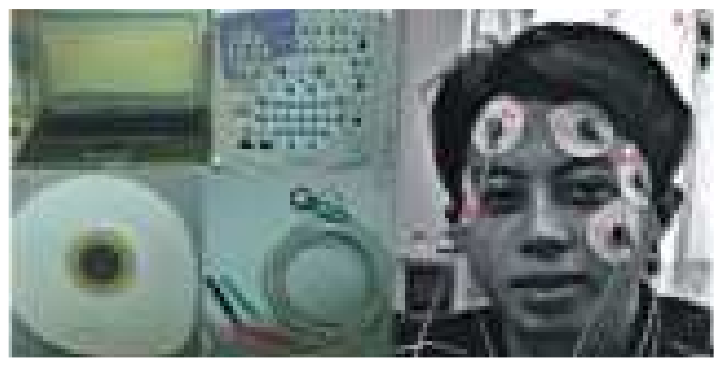

Fig. 1: (1) EEG Data Acquisition System (DAS); Neurofax 9200 software, (2) Electrode junction box to computer, (3) $\mathrm{Ag} / \mathrm{AgCl}$ electrodes, (4) Electrode connector set, (5) Subject and electrodes placement

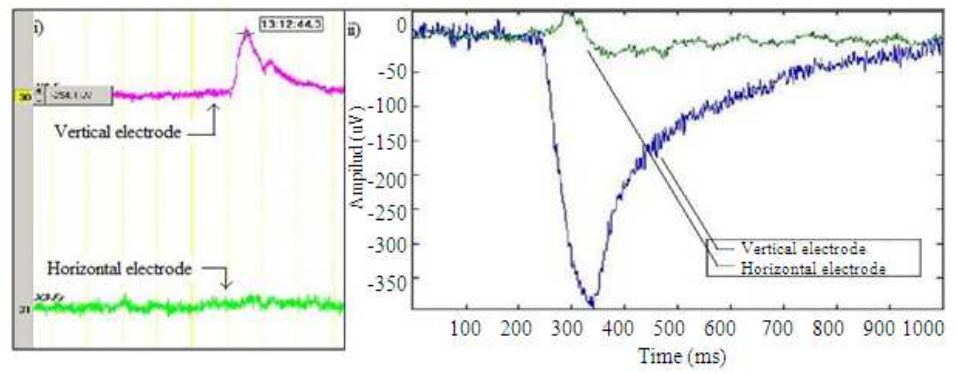

Fig. 2: (i) Real signal recorded from DAS-Neurofax EEG 9200, (ii) Signal plotted using MATLAB

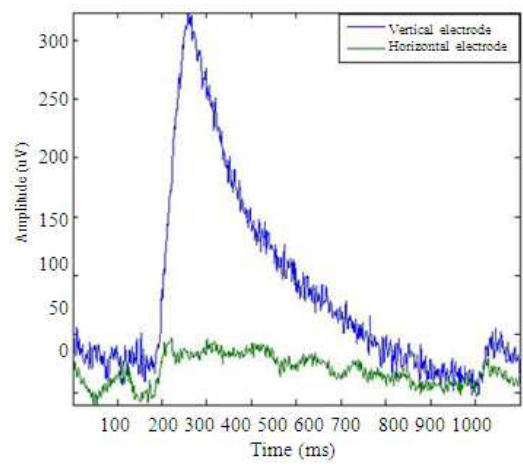

(a)

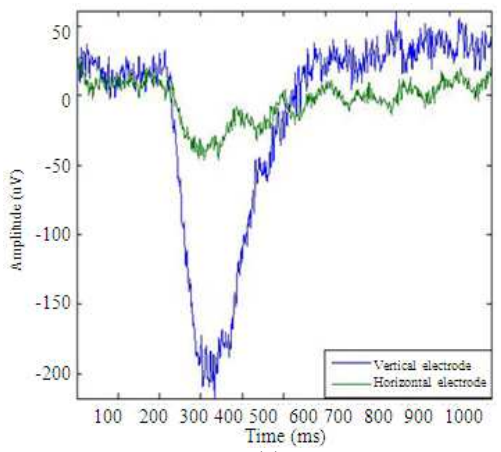

(c)

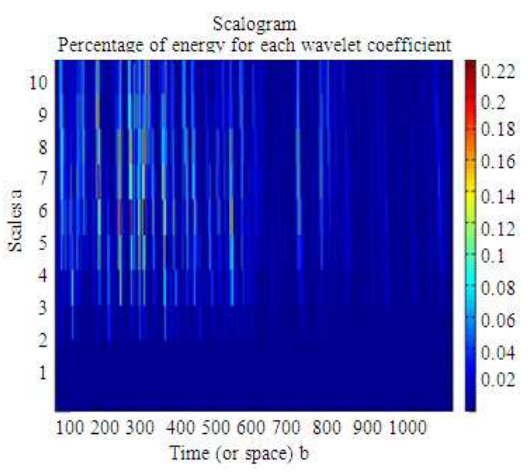

(b)

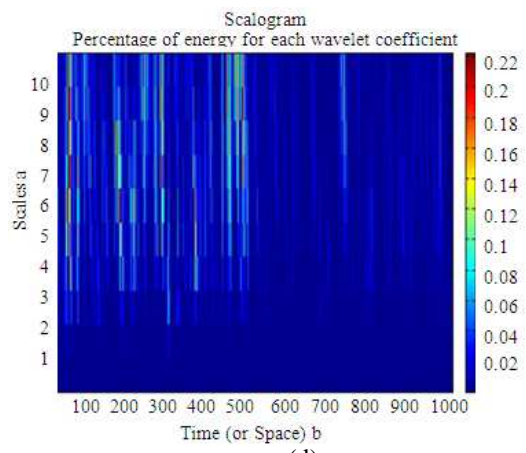

(d) 


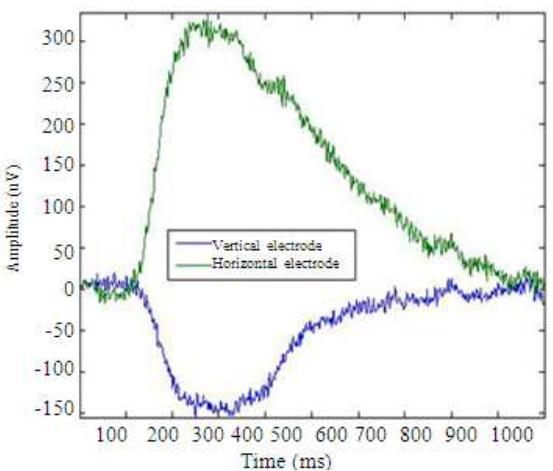

(e)

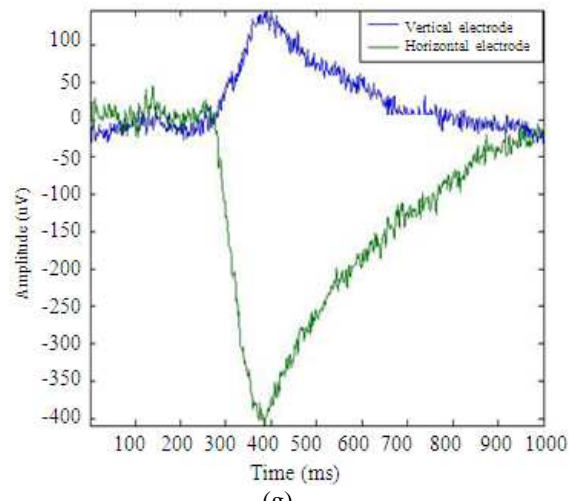

(g)
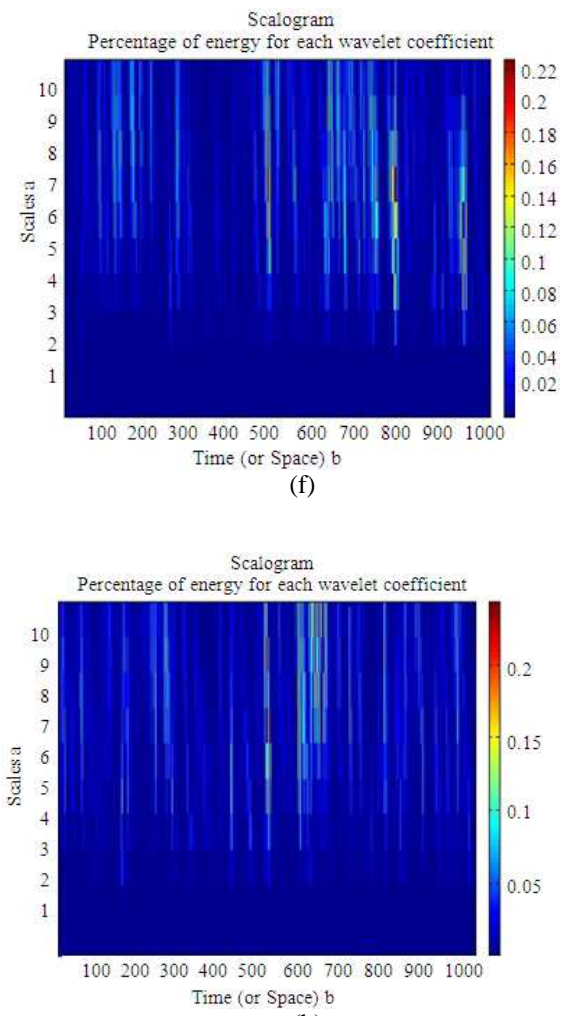

Fig. 3: (a) Downward EOG signal, (b) Upward EOG signal, (c) Leftward EOG signal and (d) Rightward EOG signal ; with their corresponding energy distributions

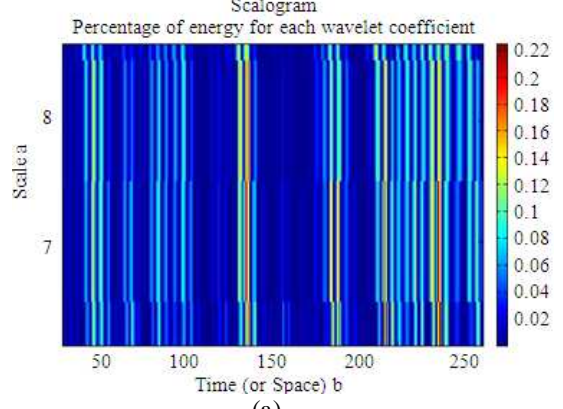

(a)

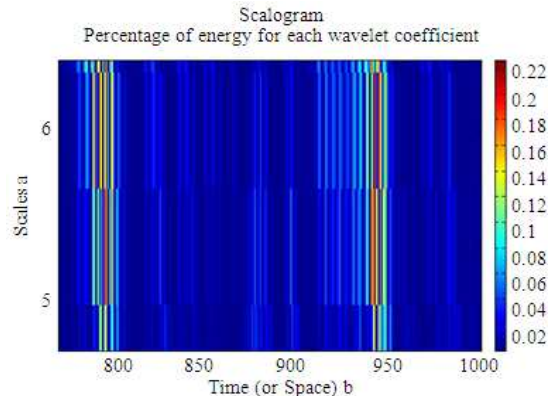

(c)

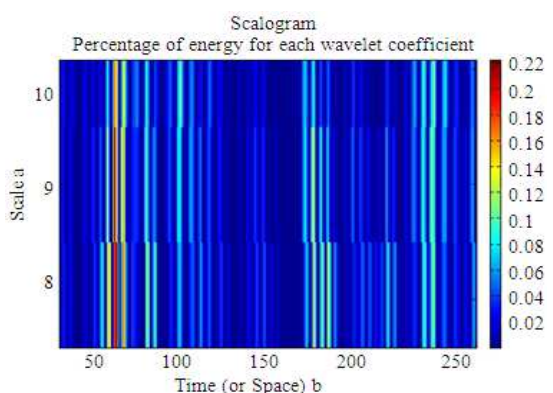

(b)

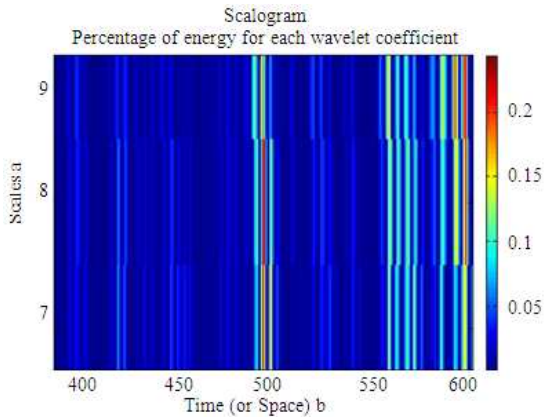

(d)

Fig. 4: Zoomed energy distribution plot for each EOG signals, (I) Downward, (II) Upward, (III) Leftward and (IV) Rightward- each signals is having their own characteristic of energy distribution 
EOG signal processing: From (Fig. 2) EOG signal captured was then analyzed by using MATLAB software and toolbox application. It was then uploaded into a program that runs a wavelet Scalogram in order to present the signal in the wavelet coefficient energy in scale and space or time. The signals is decomposed down to 10 details level by Suhartono et al. (2010) using Daubechies 4 (db4) as a mother wavelet as demonstrated by the Magosso et al. (2009). The db4 has been chosen because it has two vanishing moment i.e. constant and linear component. The numbers of level decomposition strictly depend on the sample rate of original signal, in this case we choose ten level decomposition.

\section{RESULTS AND DISCUSSION}

Pre processing: The recorded signals data in ASCII data for every eye movement and being plotted by using MATLAB as shown in (Fig. 2). Hence, the characteristic shown after plotting by using the programming in MATLAB was used to identify eye movement characteristic. Higher value is achieved when eyes move downward and lower value is achieved when eyes move to upward. Since the domain signals for the vertical fashion movement shown on vertical electrodes, the horizontal electrodes is negligible. Similar would be the case for the right-left movement. It is summarized in Table 1.

Post processing: When we come to the time frequency analysis in wavelet transform, we want to know the distribution of signal energy of wavelet details coefficient with the change of time. Hence, we plotted a Scalogram for each movement in order to identify the dominant scales with maximum wavelet energy coefficient for the signal. Scalogram is used because it represents the time frequency localization property of wavelet transform. In this plot, each details coefficient is plotted as a filled rectangle whose colors correspond to the magnitude of the coefficient.

The bar on the Scalogram plot indicates the range of energy for each level. This energy is defined as the sum of the squares of the details coefficient for each level. Figure 3A-D shows the Scalogram signals of four different movements from a subject; downward, upward, left and right from the Scalogram, it reveals that highest energy of the signals is captured in the different level of details coefficient for different EOG signals. Frequency component extracted by details move from high frequencies to low frequencies as scale of wavelet coefficient increases from 1 to 10 , with frequency content being halved at each increment in accordance to the sampling rate which is $1000 \mathrm{~Hz}$ (refer to Table 3).

About 15 data for each eye movements have been analyzed by using wavelet Scalogram in order to extract the most dominant energy details coefficient and it frequencies as shown in Fig. 4. From the (Fig. 4) it is noticed that average percentage for each movement data is slightly different from their energy level. This means that different eye movements are associated with different frequency bands. The percentage of each detail coefficient of four eye movements is derived from the resulted mean of the details coefficients. It has been concluded in the Table 2.

Statistically, from Table 2, we could decide that the dominant energy is: scale 6 for left eye movement; scale 7 for upward; scale 8 for right and scale 9 for downward. Hence, with the specific algorithm, we can derive the frequency band for each movements corresponding to their specific scales. We also calculated their accuracy of 15 data tested. It is summarized in Table 3.

Table 1: Average mean for 15 data movement

\begin{tabular}{lll}
\hline Eye Moves/Electrodes & Vertical $(\mathrm{X} 1)$ & Horizontal $(\mathrm{X} 2)$ \\
\hline Downward & Max. mean $(+\mathrm{ve})=285 \mu \mathrm{V}$ & $*$ \\
Upward & Min. mean (-ve) $=-185 \mu \mathrm{V}$ & $*$ \\
Left & $*$ & Max. mean $(+\mathrm{ve})=223 \mu \mathrm{V}$ \\
Right & $*$ & Min. mean $(-\mathrm{ve})=-382 \mu \mathrm{V}$ \\
\hline
\end{tabular}

Table 2: Means of 15 data power energy distribution for each scales

\begin{tabular}{lllll}
\hline & Means of 15 data power energy distribution for each scale (\%) & & \\
Eyes movement & 6 & 7 & 8 & 9 \\
\hline Downward & 0.11800 & 0.11357 & 0.149333 & 0.2547 \\
Upward & 0.15400 & 0.30533 & 0.138571 & 0.1313 \\
Rightward & 0.07400 & 0.14267 & 0.270000 & 0.1353 \\
Leftward & 0.24667 & 0.11333 & 0.098667 & 0.1013 \\
\hline
\end{tabular}

Table 3: Dominant energy scales and their accuracies with 15 EOG data for each movement

\begin{tabular}{lllc}
\hline EOG signal & Dominant energy scale & Accuracy $(\%)$ & Estimated frequency \\
\hline Left & Scale 6 & 80.0 & $8-16 \mathrm{~Hz}$ \\
Right & Scale 8 & 98.2 & $2-4 \mathrm{~Hz}$ \\
Up & Scale 7 & 99.2 & $4-8 \mathrm{~Hz}$ \\
Down & Scale 9 & 95.4 & $1-2 \mathrm{~Hz}$ \\
\hline
\end{tabular}


Dominant energy level means the maximum details coefficient energy that can be derived by Scalogram for each signal. We used this parameter as the benchmark to classify the different movement of EOG signals. In the study that have been done by the Bhandari et al. (2006), they used the wavelet Scalogram decomposition to determine the most energy in specific frequency bands of vertical eye movement whereas they only gave the percentage of the signal energy (90\%) of EOG signals are concentrated in the lower or higher scales and signal denoising, but they only looks for the overall wavelet decomposition and not specifically into the details of each EOG signals of each frequency bands. Hence, this study is proposed and targets the researchers to look in details of the energy and frequency bands distribution within four eye movement signals for better interpretation of EOG signals analysis by using wavelet scalogram. Result obtained indicates that each eye movement has different frequency bands.

\section{CONCLUSION}

The wavelet transform is a key time-frequency analysis and coding tool for biomedical signals. It acts like mathematical microscope, zooming into small scales to reveal compactly time-spaced events. This kind of approaches offers a lot of advantages in detecting transient features of physiological or clinical significance from bio-signals, that cannot be seen by using traditional methods such as FFT. The energetic approach built within the multi resolution decomposition and associated with a suitable chosen of mother wavelet and the number of details level, it is a powerful tool for investigation and identification of the characteristic in the bio-signals such as EOG.

In conclusion, the proposed study method could be used for developing a system for the EOG signals interpretation by using the signals energy distribution of wavelet coefficient. This study enhances the analysis of the EOG signals in giving the details of each frequency bands rely on each signal involved. The benefit of using signal energy of wavelet details coefficient is to make a precise decision in EOG signals identification for future enhancement.

\section{ACKNOWLEDGEMENT}

The authors are so indebted and would like to express our thankfulness to the University Technology Malaysia and Ministry of Science and Innovation Malaysia for supporting and funding this study. Our appreciation also goes to the Biomedical Instrumentation and Electronics Research Group (BMIE) for their ideas and comments to improve this study.

\section{REFERENCES}

Bhandari, A., V. Khare, M. Trikha and A. Anand, 2006. Wavelet based novel technique for signal conditioning of electro-oculogram signals. Proceeding of the Annual Indian Conference, Sept. 15-17, IEEE Xplore Press, New Delhi, pp: 1-6. DOI: 10.1109/INDCON.2006.302851

Choo, Y.T. and Somnuk, P.A., 2009. Log data approach to acquisition of optimal bayesian learner model. Am. J. Applied Sci., 6: 913-921. DOI: 10.3844/ajassp.2009.913.921

Daubechies, I., 1988. Orthogonal bases of compactly supported wavelets. Commun. Pure Applied Math., 41: 909-996. DOI: 10.1002/cpa.3160410705

Daubechies, I., 1990. The wavelet transform: Timfrequency localization and signal analysis. IEEE Trans. Inf. Theor., 41: 961-1005. DOI: 10.1109/18.57199

Elhelw M., M. Nicolaou, A. Chung, G.Z. Yang and M.S. Atkins, 2008. A gaze-based study for investigating the perception of visual realism in simulated, scenes. ACM Trans. Applied Percept., 5: 1-20. DOI: 10.1145/1279640.1279643

Guven, A. and S. Kara, 2006. Classification of electro-oculogram signals using artificial neural network. Expert Syst. Appl., 31: 199-205. DOI: 10.1016/j.eswa.2005.09.017

Hazarika, N., J.Z. Chen, C. Tsoi and A. Sergejew, 1997. Classification of EEG signals using the wavelet transform. IEEE Trans. Sign. Proc., 59: 61-72. DOI: 10.1016/S0165-1684(97)00038-8

Jagla, F. Jergelova and M.I. Riecansky, 2007. Saccadic eye movement related potentials. Physiol. Res., 56: 707-713.

Kherlopian, A.R., J.P. Gerrein, M. Yue, K.E. Kim and J.W. Kim et al., 2006. Electrooculogram based system for computer control using a multiple feature classification model. Conf. Proc. IEEE Eng. Med. Biol. Soc., 1: 1295-1298.

Kumar, D. and E. Poole, 2002. Classification of EOG for human computer interface. Proceedings of the Second Joint Engineering in Medicine and Biology, 2002. 24th Annual Conference and the Annual Fall Meeting of the Biomedical Engineering Society EMBS/BMES Conference, Oct. 23-26, IEEE Xplore Press, USA, pp: 64-37. DOI: 10.1109/IEMBS.2002.1134390

Lusted, H.S., R.B. Knapp and A. Lloyd, 1993. Biological signal processing in virtual reality applications. Proceedings of the 3rd Annual Virtual Reality Conference and Exhibition on VR becomes a Business, (C1993), Meckler Corporation Westport, CT, USA, pp: 101-103. 
Magosso, E., M. Ursino, A. Zaniboni and E. Gardella, 2009. A wavelet based energetic approach for the biomedical signals: Application to the EEG and EOG. Applied Math. Comput., 207: 42-62. DOI: 10.1016/j.amc.2007.10.069

Malmivuo, J. and R. Plonsey, 1995. Bioelectromagnetism: Principles and Applications of Bioelectric and Biomagnetic Fields. Oxford University Press, New York, ISBN: 0195058232, pp: 482.

Rahmat, M.F., M.D. Isa, K. Jusoff, T.A. Hussin and S.M. Rozali, 2010. Image reconstruction algorithm for electrical charge tomography system. Am. J. Applied Sci., 7: 1254-1263. DOI: 10.3844/ajassp.2010.1254.1263

Rizon, M., 2010. Discrete wavelet transform based classification of human emotions using electroencephalogram signals. Am. J. Applied Sci., $\quad 7$ : $\quad 878-885$. DOI: 10.3844/ajassp.2010.878.885
Rushaidin, M.M., S.H. Salleh, T.T. Swee, J.M. Najeb and A. Arooj, 2009. Wave V detection using instantaneous energy of auditory brainstem response signal. Am. J. Applied Sci., 6: 16691674. DOI: 10.3844/ajassp.2009.1669.1674

Shinomiya, K., N. Itsuki, M. Kubo and H. Shiota, 2008. Analyses of the characteristics of potential and cross-talk at each electrode in Electrooculogram. J. Med. Invest., 55: 120-126. DOI: 10.2152/jmi.55.120

Sudirman, R. and W.M. Bukhari, 2009. Classification of eye movement potentials using time frequency analysis. Proceedings of the 13th IASTED International Conference on Signal and Image Processing Sip, Dec. 14-16, ACTA Press, USA, pp: 131-135.

Suhartono, B. S.S. Ulama and A. J. Endharta, 2010. Seasonal time series data forecasting by using neural networks multiscale autoregressive model. Am. J. Applied Sci., 7: 1372-1378. DOI: 10.3844/ajassp.2010.1372.1378 